\title{
Cardiac stunning as first manifestation of multiple sclerosis: A case report reminding us not to overlook cardiovascular autonomic dysfunction in multiple sclerosis
}

\author{
Max J Hilz
}

Abstract: Autonomic dysfunction is common but frequently overlooked in multiple sclerosis (MS) patients. The case of a Tako-Tsubo cardiomyopathy on which this commentary is based shows that centrally triggered autonomic dysfunction may be the first life-threatening manifestation of MS.

Keywords: Multiple sclerosis, cardiovascular autonomic dysfunction, Tako-Tsubo cardiomyopathy

Autonomic dysfunction is known to contribute to disability in multiple sclerosis (MS) patients. ${ }^{1}$

Up to $84 \%$ of MS patients experience dysfunction of autonomic organ regulation. ${ }^{1}$ Common complaints include bowel and bladder disturbances, sexual dysfunction, or sweating abnormalities. ${ }^{1,2}$ Cardiovascular autonomic dysfunction may include baroreflex dysfunction with orthostatic intolerance and orthostatic hypotension, postural tachycardia syndrome, or decreased heart rate variability ${ }^{3}$ which is associated with an increased risk of cardiovascular complications and reduced life expectancy.,34 Although autonomic dysfunction frequently impairs the patient's quality of life, ${ }^{3}$ autonomic dysregulation and its prognostic impact seem to be poorly addressed in the clinical evaluation of MS patients. The most common scales evaluating MS severity, the Expanded Disability Status Scale $(\mathrm{EDSS})^{5}$ and Multiple Sclerosis Functional Composite (MSFC), ${ }^{6}$ largely ignore the clinical and prognostic relevance of autonomic dysfunction.

This is remarkable since the autonomic nervous system is not only afflicted by the neuro-inflammatory process but also contributes to maintaining inflammation and disease progression. ${ }^{7}$ MS-induced changes in sympathetic or parasympathetic modulation may in turn alter immune responses. ${ }^{7}$

Particularly during the first years after disease manifestation, MS patients seem to have an increased risk of cardiovascular diseases, ${ }^{8}$ which is very likely to be due to a central up-regulation of sympathetic outflow to the cardiovascular system. ${ }^{7}$
Still, acute cardiovascular emergencies are rarely recognized as initial manifestation of MS, probably because cardiologists and neurologists are unaware of this etiology.

However, sympathetic activity may increase significantly during early stages of MS, while later relapses may lead to progressive noradrenergic failure and adrenergic receptor dysfunction. ${ }^{7}$ In stroke patients, neurologists are increasingly aware of the risk of abundant cardiovascular autonomic disorders ${ }^{9}, 10$ In contrast, acute cardiologic emergencies might not be recognized as centrally mediated in patients who experience such an emergency, and supposedly by coincidence, also happen to have MS.

In this issue of the Multiple Sclerosis Journal (MSJ), Madaglia et al. present the case of a previously healthy young man who was admitted to internal medicine because of severe headache and diaphoresis and a first-degree atrioventricular block. ${ }^{11}$ After 2 weeks, symptoms reoccurred but were more severe, with additional tachycardia and dyspnea, arterial hypertension, and electrocardiographic changes that are typical for stress-cardiomyopathy, ${ }^{12}$ also called Tako-Tsubo cardiomyopathy (TTC). TTC may be life threatening and is characterized by apical ballooning, left ventricular dysfunction with ventricular wallmotion abnormalities. ${ }^{12,13}$ Although the pathogenesis of TTC is not yet fully understood, ${ }^{12}$ there is a dysfunction in the brain-heart axis, most likely with increased sympathetic activity and catecholamine surges. ${ }^{13}$ The patient had no myocarditis or acute myocardial infarction but turned out to have MS with
Multiple Sclerosis Journal 2016, Vol. 22(6) 847-848 DOI: $10.1177 /$ 1352458516638559

(c) The Author(s), 2016. Reprints and permissions: http://www.sagepub.co.uk/ journalsPermissions.nav

\author{
Correspondence to: \\ MJ Hilz \\ Department of \\ Neurology, University \\ of Erlangen-Nuremberg, \\ Schwabachanlage 6, D-91054 \\ Erlangen, Germany. \\ max.hilz@uk-erlangen.de \\ Max J Hilz \\ Department of Neurology, \\ University of Erlangen- \\ Nuremberg, Erlangen, \\ Germany
}


several active lesions, some of which involved areas of the central autonomic network, for example, the medulla oblongata. Methylprednisolone treatment quickly reversed the myocardial stunning.

The case alerts neurologists to the fact that MS may manifest as cardiovascular emergency. ${ }^{3}$ Although it seems to be the first report of TTC as the initial symptom of MS, cardiovascular complications of MS are frequent and range from subclinical to life-threatening events and may cause fatalities that have been coined as "SUDIMS," Sudden Unexpected Death in MS, ${ }^{3}$ in analogy to "SUDEP," the Sudden Unexpected Death in Epilepsy. ${ }^{14}$

In summary, the case reminds us to pay closer attention to centrally mediated autonomic dysfunction in MS patients and to better investigate the association between neuro-inflammation and cardiovascular autonomic dysfunction.

\section{Declaration of Conflicting Interests}

The author(s) declared no potential conflicts of interest with respect to the research, authorship, and/or publication of this article.

\section{Funding}

The author(s) received no financial support for the research, authorship, and/or publication of this article.

\section{References}

1. Racosta JM, Kimpinski K, Morrow SA, et al. Autonomic dysfunction in multiple sclerosis. Auton Neurosci 2015; 193: 1-6.

2. Merkelbach S, Haensch CA, Hemmer B, et al. Multiple sclerosis and the autonomic nervous system. J Neurol 2006; 253(Suppl. 1): I21-I25.
4. Scalfari A, Knappertz V, Cutter G, et al. Mortality in patients with multiple sclerosis. Neurology 2013; 81 : 184-192.

5. Kurtzke JF. Rating neurologic impairment in multiple sclerosis: An expanded disability status scale (EDSS). Neurology 1983; 33: 1444-1452.

6. Cutter GR, Baier ML, Rudick RA, et al. Development of a multiple sclerosis functional composite as a clinical trial outcome measure. Brain 1999; 122(Pt 5): 871-882.

7. Racosta JM and Kimpinski K. Autonomic dysfunction, immune regulation, and multiple sclerosis. Clin Auton Res 2016; 26: 23-31.

8. Christiansen CF. Risk of vascular disease in patients with multiple sclerosis: A review. Neurol Res 2012; 34: 746-753.

9. Hilz MJ and Schwab S. Stroke-induced suddenautonomic death: Areas of fatality beyond the insula. Stroke 2008; 39: 2421-2422.

10. Soros P and Hachinski V. Cardiovascular and neurological causes of sudden death after ischaemic stroke. Lancet Neurol 2012; 11: 179-188.

11. Midagliaa L, Juega Mariñob JM, Sastre-Garrigaa J, et al. An uncommon first manifestation of multiple sclerosis: Tako-Tsubo cardiomyopathy. Mult Scler 2016; 22: 842-846.

12. Templin C, Ghadri JR, Diekmann J, et al. Clinical features and outcomes of takotsubo (stress) cardiomyopathy. N Engl J Med 2015; 373: 929-938.

13. Prasad A, Lerman A and Rihal CS. Apical ballooning syndrome (Tako-Tsubo or stress cardiomyopathy): A mimic of acute myocardial infarction. Am Heart $J$ 2008; 155: 408-417.

14. Dütsch M and Hilz MJ. Epilepsy and autonomic regulation. In: Robertson D, Biaggioni I, Burnstock $\mathrm{G}$, et al. (eds) Primer on the autonomic nervous system. San Diego, CA: Academic Press; Elsevier, 2012, pp. 549-552. 